\title{
NUMERICAL ANALYSIS OF THE INFLUENCE OF A NEARBY FIBRE ON THE INTERFACE CRACK GROWTH UNDER TRANSVERSE TENSILE LOAD
}

\author{
C. Sandino*, E. Correa ${ }^{* *}$, F. París \\ Elasticity and Strength of Materials Group, School of Engineering, University of Seville, \\ Camino de los Descubrimientos s/n, 41092 Seville, Spain \\ *carsandeb@alum.us.es;**ecorrea@us.es
}

Keywords: Micromechanics, Interfacial Fracture Mechanics, Matrix/inter-fibre failure, Twofibres model.

\begin{abstract}
In this paper the interface crack growth associated to the inter-fibre failure under tension is studied by means of a two-fibres BEM model. The influence of the presence of an undamaged secondary fibre on the evolution of the interface crack at a primary one is analysed by means of Interfacial Fracture Mechanics concepts. The results obtained show that when the secondary fibre is aligned with the direction of the external load, its presence has an accelerative effect versus interface crack growth, whereas it has a protective effect for the rest of the positions considered.
\end{abstract}

\section{Introduction}

The mechanism of damage known as matrix/inter-fibre failure under uniaxial tension has already been the object of several studies by the authors for the single fibre case, [1-4]. Based on the hypothesis that transverse failure starts with the appearance of small debonds at the fibre-matrix interfaces, the studies presented in [1-4] allowed identifying the subsequent scheme, which defines the stages of the inter-fibre failure under uniaxial tension:

1. Crack nucleation: it is assumed to be controlled by the radial stress that appears between fibre and matrix. In the single fibre case, the maximum values of the stress are detected at the angles $0^{\circ}$ and $180^{\circ}$ in relation to the tension applied.

2 . Interface crack growth: the crack grows symmetrically and in an unstable way along the interface until it reaches a certain length $\left(60-70^{\circ}\right)$, coinciding with the appearance of a finite contact zone at the tip.

3. Kinking: the interface crack suddenly modifies its growth direction, kinking into the matrix in the direction perpendicular to the load applied. The coalescence between different cracks in the matrix leads to the final macro-failure.

In this study, focusing on the second stage of the mechanism of damage, a two-fibres BEM model is developed in order to evaluate the effect of the presence of an undamaged secondary nearby fibre on the evolution of an interface crack existent at a central primary one. Interfacial Fracture Mechanics concepts [5] are used for the analysis of the results obtained. Previous studies focusing on different aspects of two-fibres models have already been developed by several authors, using FEM [6, 7] and analytical approaches [8]. 
Thus, the objective of this paper is, considering different positions of the secondary fibre, to evaluate the effect of the nearby fibre on the matrix/inter-fibre failure; the study is focused on crack morphology and propagation, following the approach already presented in [9].

\section{Numerical model}

The numerical study has been carried out using a tool based on BEM [10], that makes it possible to perform the numerical analysis of plane elastic problems considering contact and interface cracks, in a similar way to that described in [11] for planar problems and [12] for axisymmetric problems. The basic model employed is shown in Fig. 1 and represents the case of a crack which, under the plane strain hypothesis, grows along the interface symmetrically to axis $\mathrm{x}$ and in presence of a nearby fibre.

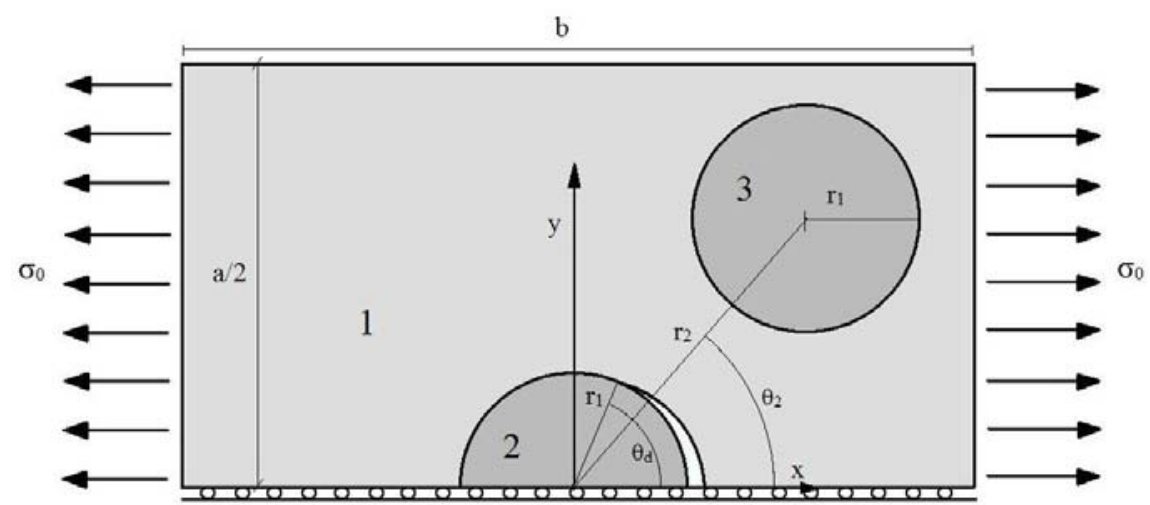

Figure 1. Model including interface crack and a secondary fibre.

Solid 1 represents the matrix and Solids 2 and 3 the primary and secondary fibres respectively. The fibres dimensions are defined by the radius $r_{l}$ and the matrix dimensions are large enough in order to avoid the border effect. Specifically, the conditions $b=100 \cdot r_{1}$ and $a=b / 2$ are imposed. The materials chosen for the analysis correspond to a glass fibre-epoxy matrix system. The elastic properties are included in Table 1:

\begin{tabular}{ccc}
\hline Material & Young modulus, $\boldsymbol{E}[\mathrm{Pa}]$ & Poisson coefficient, $\boldsymbol{v}$ \\
Matrix (epoxy) & $E^{m}=2.79 \cdot 10^{9}$ & $v^{m}=0.33$ \\
Fibre (glass) & $E^{f}=7.08 \cdot 10^{10}$ & $v^{f}=0.22$ \\
\hline
\end{tabular}

Table 1. Elastic properties of the materials.

The fibre radius considered is $r_{I}=7.5 \cdot 10^{-6} \mathrm{~m}$ and the position of the secondary fibre, defined by the parameters $r_{2}$ and $\theta_{2}$, is changed in order to generate the considered cases. For this study the initial distance employed between the fibres is $r_{2}=r_{2}^{0}=2.416 \cdot r_{1}$, which corresponds to a fibre volume fraction of $62 \%$ (assuming hexagonal packing).

To characterise the problem from the Fracture Mechanics point of view the Energy Release Rate, $G$, is used. The expression employed, based on [13], for a circular crack that propagates from a certain debonding angle, $\theta_{d}$, Fig. 1 , to $\theta_{d}+\Delta \theta_{d}\left(\Delta \theta_{d}<<\theta_{d}\right)$, is:

$$
G\left(\theta_{d}, \Delta \theta_{d}\right)=\frac{1}{2 \Delta \theta_{d}} \int_{0}^{\Delta \theta_{d}}\left[\sigma_{r r}\left(\theta_{d}+\theta\right) \Delta u_{r}\left(\theta_{d}-\Delta \theta_{d}+\theta\right)+\sigma_{r \theta}\left(\theta_{d}+\theta\right) \Delta u_{\theta}\left(\theta_{d}-\Delta \theta_{d}+\theta\right)\right] d \theta
$$

where $\theta$ is the circumferential coordinate with reference to axis $\mathrm{x} . \sigma_{r r}$ and $\sigma_{r \theta}$ represent, respectively, radial and shear stresses along the interface, and $\Delta u_{r}$ and $\Delta u_{\theta}$ represent the 
relative displacements of the crack faces. Both modes of fracture, I (associated to $\sigma_{r r}$ ) and II, (associated to $\sigma_{r \theta}$ ), are obviously considered in Eq. (1). For this study the value of $\Delta \theta_{d}$ employed has been $0.5^{\circ}$.

Dimensionless results for $G$ will be presented. These values are obtained, following [14,15], by dividing the values of $G$ by $G_{0}$ :

$$
G_{0}=\left(\left(1+\kappa^{m}\right) 8 \mu^{m}\right) \sigma_{0}^{2} r_{1} \pi
$$

where $\kappa^{m}=3-4 v^{m}, \mu^{m}$ is the shear modulus of the matrix and $\sigma_{0}$ is the applied external tension.

\section{Energy Release Rate}

The $G$ distributions versus the debonding angle of the crack $\left(10^{\circ} \leq \theta_{d} \leq 150^{\circ}\right)$ are calculated from Eq. (1) and compared by changing the position of the secondary fibre $\left(25^{\circ} \leq \theta_{2} \leq 155^{\circ}\right)$. A selection of these distributions is represented in Fig. 2. The reference case (single-fibre) is included in the figure.

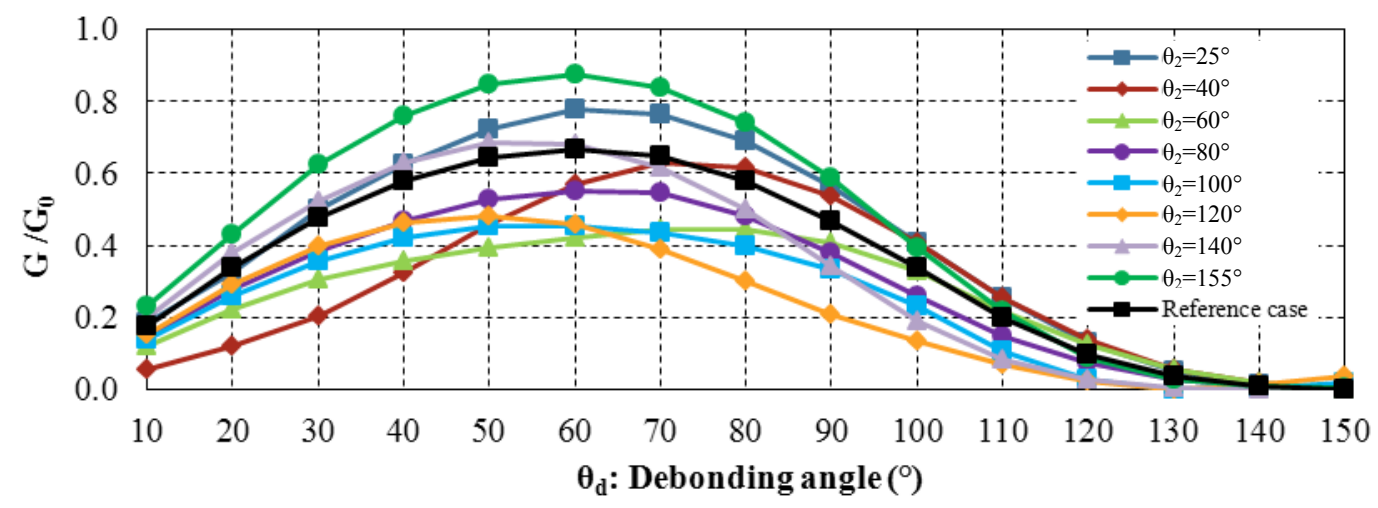

Figure 2. $G / G_{0}$ versus $\theta_{d}$.

Curves presented in Fig. 2 show that, when the secondary fibre is close to the symmetry plane $\left(25^{\circ} \leq \theta_{2} \leq 40^{\circ}\right.$ and $\left.140^{\circ} \leq \theta_{2} \leq 155^{\circ}\right)$ and with reference to the single fibre model, the $G$ values are total or partially above the reference energetic distribution. Nevertheless, the presence of a secondary fibre for the rest of the positions (intermediate ones) produces the opposite effect, since the curves reach lower values than the reference model. This $G$ decrement is more significant for $\theta_{2}=60^{\circ}$ and $100^{\circ} \leq \theta_{2} \leq 110^{\circ}$. The smallest decrement is produced for $\theta_{2}=80^{\circ}$.

In particular and with reference to the $G$ value associated to the first debond considered $\left(\theta_{d}=10^{\circ}\right)$, it is observed that for $\theta_{2}=25^{\circ}$ and $140^{\circ} \leq \theta_{2} \leq 155^{\circ}$, the $G$ distributions are above the reference case one. For the rest of the positions, the initial $G$ values are below that associated to the reference case.

Moreover, with reference to the qualitative distribution of the curves, another effect is observed. In several cases, the position of the maximum values changes. In particular, the maxima of the curves are reached for higher debonding angles in the $30^{\circ} \leq \theta_{2} \leq 60^{\circ}$ cases than in the reference case, whose maximum occurs for $\theta_{d}=60^{\circ}$, whereas in the $110^{\circ} \leq \theta_{2} \leq 140^{\circ}$ cases, the maxima are reached for lower debonding angles.

In Figs. 3 and 4, Mode I and II components $\left(G_{I}\right.$ and $\left.G_{I I}\right)$ are represented versus $\theta_{d}$ for different values of $\theta_{2}$; the reference case is also included. It is observed that the evolution of the 
interface crack follows the subsequent stages: the crack starts growing under Mode I, later, it propagates under mixed mode and finally it grows under pure Mode II. As happened with the global $G$ (Fig. 2), the $G_{I}$ and $G_{I I}$ distributions remain partially or totally above the reference distribution when the fibre is close to the symmetry plane and below it for the rest of the positions.

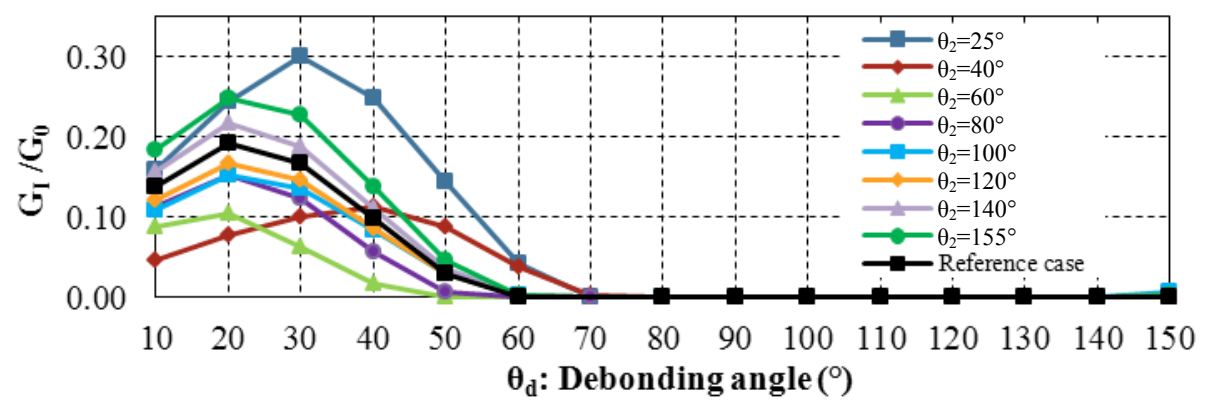

Figure 3. $G_{I} / G_{0}$ versus $\theta_{d}$.

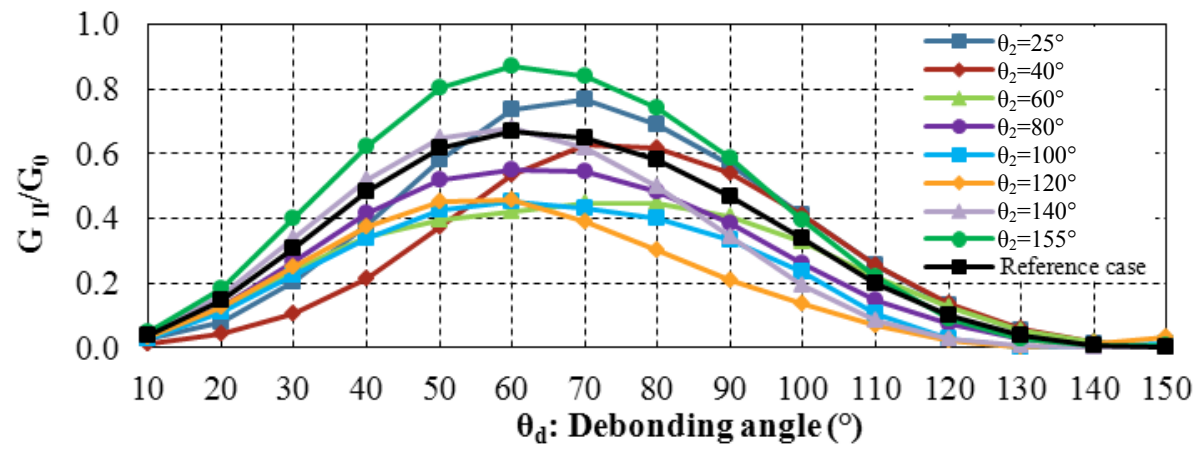

Figure 4. $G_{I I} / G_{0}$ versus $\theta_{d}$.

The $G_{I}$ distributions, Fig. 3, present significant differences for $25^{\circ} \leq \theta_{2} \leq 40^{\circ}$, with reference to the single fibre case. For this interval, the values of $G_{I}$ change abruptly, in terms of the energy level. With regard to the position of the maxima, for the $50^{\circ} \leq \theta_{2} \leq 155$ cases it occurs at $\theta_{d}=20^{\circ}$, coinciding with the reference case, whereas for the $25^{\circ} \leq \theta_{2} \leq 40^{\circ}$ cases it takes place at higher debonding angles.

In the $\mathrm{G}_{I I}$ distributions, Fig. 4 , the maxima are reached at $\theta_{d}=60^{\circ}$ for the reference case and most of the positions considered. However, for $25^{\circ} \leq \theta_{2} \leq 60^{\circ}$, the maxima take place at larger values of $\theta_{d}$.

\section{Fracture mode mixity}

Due to its importance for the determination of the damage evolution, the change of crack propagation from mixed mode to pure Mode II of fracture is carefully observed, even though there are not major variations on the debonding angle for which Mode I disappears. Table 2 shows the values obtained for the different secondary fibre positions considered.

\begin{tabular}{|c|c|c|c|c|c|}
\hline$\theta_{2}\left[^{\circ}\right]$ & $\theta_{d\left(\text { mixed } \_I I\right)}\left[{ }^{\circ}\right]$ & $\theta_{2}\left[^{\circ}\right]$ & $\theta_{d\left(\text { mixed } \_I I\right)}\left[^{\circ}\right]$ & $\boldsymbol{\theta}_{2}\left[^{\circ}\right]$ & $\theta_{d(\text { mixed_II })}\left[^{\circ}\right]$ \\
\hline 25 & 70 & 70 & 50 & 120 & 60 \\
\hline 30 & 70 & 80 & 50 & 130 & 60 \\
\hline 40 & 70 & 90 & 60 & 140 & 60 \\
\hline 50 & 60 & 100 & 60 & 150 & 60 \\
\hline 60 & 50 & 110 & 60 & 155 & 60 \\
\hline
\end{tabular}

Table 2. Debonding angle $\left(\theta_{d}\right)$, for which Mode I disappears for each secondary fibre position $\left(\theta_{2}\right)$. 
According to Figs. 3 and 4 , for $25^{\circ} \leq \theta_{2} \leq 40^{\circ}, G_{I I}$ becomes dominant versus $G_{I}$ at $\theta_{d} \approx 30-40^{\circ}$, i.e. later than in the single fibre model $\left(\theta_{d} \approx 25^{\circ}\right)$. Moreover, in the reference case, Mode I disappearance is detected for $\theta_{d} \approx 60^{\circ}$ whereas for the $25^{\circ} \leq \theta_{2} \leq 40^{\circ}$ cases it occurs for larger debonding angles, specifically at $\theta_{d} \approx 70^{\circ}$. Nevertheless, for $60^{\circ} \leq \theta_{2} \leq 80^{\circ}$, an inversion of this tendency is observed, since Mode II starts to be dominant at $\theta_{d} \approx 20^{\circ}$ and finally Mode I disappears at $\theta_{d} \approx 50^{\circ}$. For the rest of secondary fibre positions, there are not significant differences with reference to the single fibre case.

\section{Crack morphology}

The morphology of the crack numerically predicted for the single fibre model shows that the finite contact zone is not developed until $\theta_{d} \approx 60^{\circ}$. Fig. 5 shows that, with reference to the single fibre case, the contact zone is slightly delayed for $25^{\circ} \leq \theta_{2} \leq 40^{\circ}\left(\theta_{d} \approx 70^{\circ}\right)$ and advanced for $60^{\circ} \leq \theta_{2} \leq 80^{\circ}\left(\theta_{d} \approx 50^{\circ}\right)$. For the rest of the positions, the development of the finite contact zone does not start until $\theta_{d} \approx 60^{\circ}$.

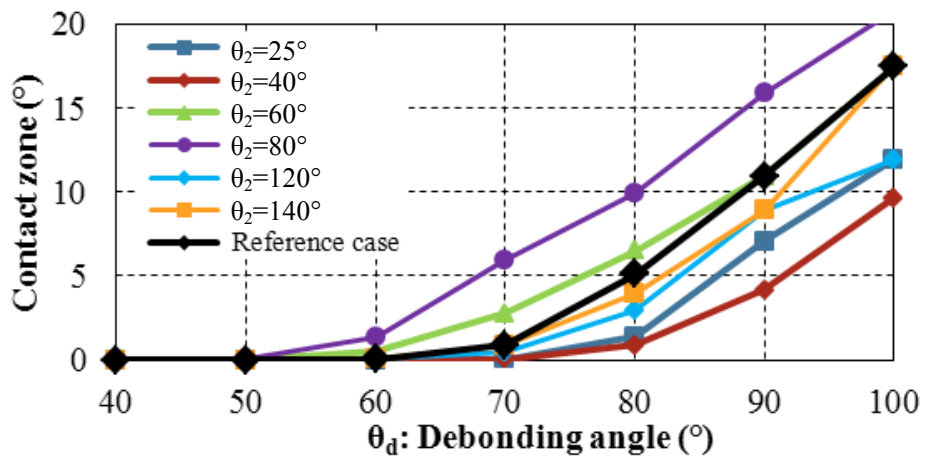

Figure 5. Contact zone evolution for different positions of the secondary fibre $\left(\theta_{2}\right)$.

For $25^{\circ} \leq \theta_{2} \leq 50$ and $110^{\circ} \leq \theta_{2} \leq 155^{\circ}$ the evolutions of the contact zone are below the reference case values, whereas for $70^{\circ} \leq \theta_{2} \leq 90^{\circ}$, they are above it. The $\theta_{2}=60^{\circ}$ and $\theta_{2}=100^{\circ}$ cases are considered as transition cases, since the detected contact zone values are close to the reference case ones.

For instance, according to Fig. 5 , the $\theta_{2}=80^{\circ}$ case, whose contact zone is advanced, produces larger lengths of contact, whereas the $\theta_{2}=40^{\circ}$ case, whose contact zone is delayed, produces smaller ones. Thus, the contact zone detected, e.g., for an interface crack of $\theta_{d}=90^{\circ}$, is almost 4 times larger for $\theta_{2}=80^{\circ}$ than for $\theta_{2}=40^{\circ}$. In order to check the results and visualise them on the crack morphology, this deformed situation is represented in Fig. 6, by amplifying the interface displacements $10^{7}$ times.

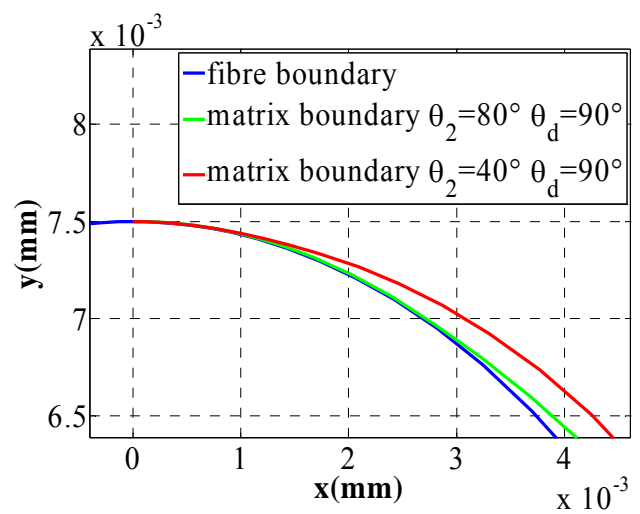

Figure 6. Effects of the secondary fibre position on the interface crack contact zone. 


\section{Propagation of the interface crack}

The predictions about the interface crack growth can be performed, as explained in [9], using the estimation of the critical value of the Energy Release Rate, $G_{c}$, based on [16]:

$$
G_{c}\left(\psi_{k}\right)=G_{1 c}\left[1+\tan ^{2}\left[(1-\lambda) \psi_{k}\right]\right]
$$

where $G_{l c}$ is the critical value of $G_{c}$ for Mode $\mathrm{I}$ and $\lambda$ is the fracture mode sensitivity parameter (in this study, $\lambda=0.25$ ). $\psi_{k}$ is the local phase angle and represents the evolution of the fracture mode mixity. It can be calculated following [17]:

$$
\psi_{k}(G)=0.5 \arccos \left[F(\varepsilon)^{-1} \frac{G_{I}-G_{I I}}{G_{I}+G_{I I}}\right]
$$

where $\varepsilon$ is the oscillatory index and $F(\varepsilon)^{-1}=1.0071$.

Fig. 7 shows the evolutions of $\psi_{k}$ and confirms the tendencies observed in Sections 4 and 5 about mixity and appearance of the contact zone.

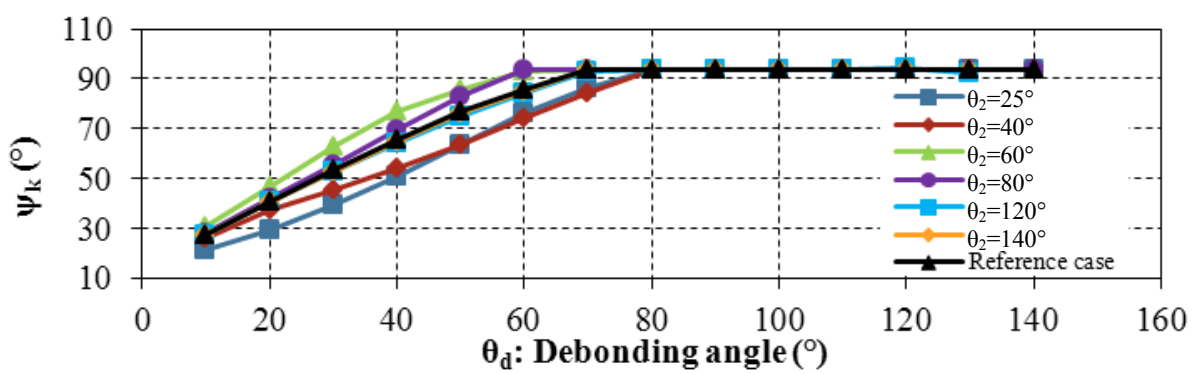

Figure 7. Local phase angle $\left(\psi_{k}\right)$ evolution for different positions of the secondary fibre $\left(\theta_{2}\right)$.

The comparison of the evolutions of $G$ and $G_{\mathrm{c}}$ predicts the ending of the unstable growth of the interface crack. This comparison is obtained using Eq. (3), where the calculation of $G_{l c}$ follows the approach employed in [9].

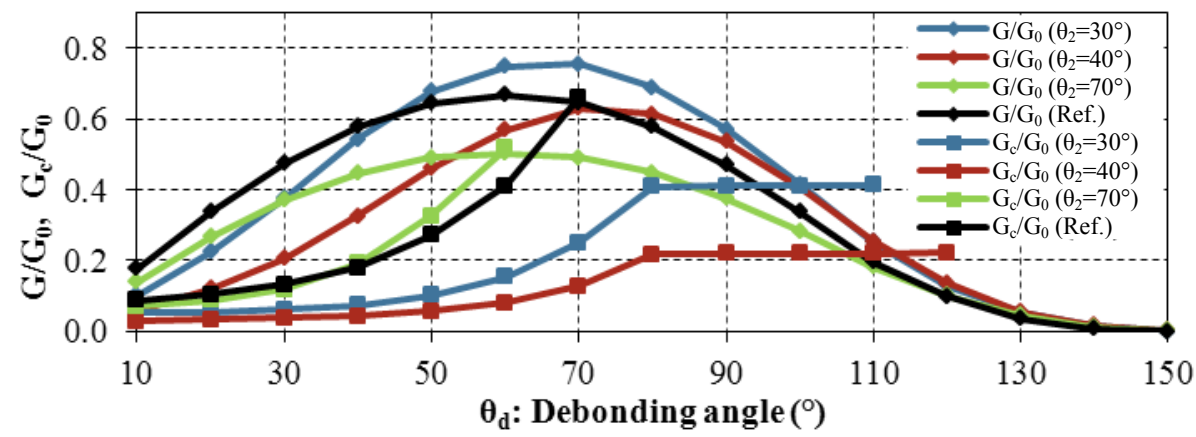

Figure 8. $G-G_{c}$ comparison for different positions of the secondary fibre $\left(\theta_{2}\right)$.

Fig. 8 shows the $G$ and $G_{\mathrm{c}}$ results obtained for different values of $\theta_{2}\left(30^{\circ}, 40^{\circ}\right.$ and $\left.70^{\circ}\right)$ and also includes the single fibre case. It can be observed that the single fibre model predicts unstable growth until $\theta_{d} \approx 70^{\circ}$. The results also show that the interface crack extends towards larger debonding angles for $\theta_{2}=25^{\circ}$ (specifically $\left.\theta_{d} \approx 80^{\circ}\right), \theta_{2}=30^{\circ}\left(\theta_{d} \approx 100^{\circ}\right), \theta_{2}=40^{\circ}\left(\theta_{d} \approx 112^{\circ}\right)$ and $\theta_{2}=50^{\circ}\left(\theta_{d} \approx 105^{\circ}\right)$, and smaller for $60^{\circ} \leq \theta_{2} \leq 90^{\circ}\left(\theta_{d} \approx 60^{\circ}\right)$. Finally, for $\theta_{2} \geq 100^{\circ}$ (not 
included in the figure), the crack growth prediction coincides with that associated to the reference case.

\section{Effect of the distance of the nearby fibre}

In order to evaluate the influence of the distance between both fibres, a change of the parameter $r_{2}$ has been carried out. This change allows verifying the previously observed tendencies and establishing an $r_{2}$ value from which the influence of the secondary fibre could be ignored.

Table 3 shows the values of $r_{2}$ and the relations $r_{2} / r_{2}{ }^{0}$ that determine the end of the influence of the secondary fibre on the different aspects of the interface crack problem studied in the previous sections. The results reveal that for the interval $1.5 \leq r_{2} / r_{2}{ }^{0} \leq 2.5$ the influence disappears for most of these aspects. However, the effects on the $G$ level, for most of the positions of the secondary fibre, remain until $r_{2} / r_{2}{ }^{0}=7$.

\begin{tabular}{|c|c|c|}
\hline Section & & $\mathbf{r}_{2} / \mathbf{r}_{2}{ }^{0}$ \\
\hline 3 & $\begin{array}{l}\text { Convergence of the } G \text { values towards the reference case } \\
\left(80^{\circ} \leq \theta_{2} \leq 90^{\circ}\right)\end{array}$ & 1.5 \\
\hline 3 & $\begin{array}{l}\text { Convergence of the } G \text { values towards the reference case } \\
\text { (rest of the positions of the secondary fibre) }\end{array}$ & 7 \\
\hline 3 & $\begin{array}{l}\text { Disappearance of the delay/advance of the position of the } G \\
\text { maximum }\end{array}$ & 2 \\
\hline 4 & Disappearance of the delay/advance of the fracture mode mixity & 2 \\
\hline 5 & Disappearance of the delay/advance of the contact zone & 2 \\
\hline 6 & Disappearance of the variation of the unstable crack growth & 2.5 \\
\hline
\end{tabular}

Table 3. Influence of the distance between the primary and secondary fibres.

\section{Conclusions}

According to the results, the most important effects on the interface crack are produced when the position of the secondary fibre lies within the interval $25^{\circ} \leq \theta_{2} \leq 90^{\circ}$. For the interval $25^{\circ} \leq \theta_{2} \leq 50^{\circ}$, the unstable growth of the interface crack stops at larger lengths of the crack than in the reference case. Nevertheless, the opposite effect is produced for the interval $60^{\circ} \leq \theta_{2} \leq 90^{\circ}$. Quantitatively, the effects for the $25^{\circ} \leq \theta_{2} \leq 50^{\circ}$ cases are more significant, since the increase of the crack growth $\left(\theta_{d} \approx 80^{\circ}-112^{\circ}\right)$, with reference to the single fibre case, is larger than the decrease detected in the $60^{\circ} \leq \theta_{2} \leq 90^{\circ}$ cases $\left(\theta_{d} \approx 60^{\circ}\right)$.

It is observed that for $\theta_{2}=25^{\circ}$ and $140^{\circ} \leq \theta_{2} \leq 155^{\circ}$, the $G$ values at $\theta_{d=10^{\circ}}$ (first debond considered) are above the reference case one, which means that a lower level of load is needed to initiate the propagation; i.e. an accelerative effect of the presence of the secondary fibre versus failure. For the rest of the positions, the $G$ values at $\theta_{d=10^{\circ}}$ are below the reference case, involving a protective effect.

With regard to the distance between the fibres, it is observed that the most important effects disappear for $r_{2} / r_{2}{ }^{0}=2.5$, although the total disappearance of the effects is produced for $r_{2} / r_{2}{ }^{0}=7$. 


\section{Acknowledgements}

This work was supported by the Spanish Ministry of Education and Science/Economy and Competitiveness and Junta de Andalucía (Projects MAT2012-37387, DPI 2012-37187, TEP4051 and TEP-7093).

\section{References}

[1] F. París, E. Correa and V. Mantič. Kinking of transverse interface cracks between fibre and matrix. J App Mech, 74: 703-716, 2007.

[2] F. París, E. Correa and J. Cañas. Micromechanical view of failure of the matrix in fibrous composite materials. Compos Sci Technol, 63: 1041-1052, 2003.

[3] E. Correa, E.K. Gamstedt, F. París and V. Mantič. Effects of the presence of compression in transverse cyclic loading on fibre-matrix debonding in unidirectional composite plies. Composites Part A, 38: 2260-2269, 2007.

[4] E. Correa, V. Mantič and F. París. Effect of thermal residual stresses on the matrix failure under transverse tension at micromechanical level. A numerical and experimental analysis. Compos Sci Technol, 71(5): 622-629, 2011.

[5] V. Mantič, A. Blázquez, E. Correa and F. París. Analysis of interface cracks with contact in composites by 2D BEM. In M. Guagliano and M.H. Aliabadi, editors. Fracture and Damage of Composites, pages 189-248. WIT Press, Southampton, 2006.

[6] V.I. Kushch, S.V. Shmegera, P. Brøndsted, L. Mishnaevsky Jr. Numerical simulation of progressive debonding in fiber reinforced composite under transverse loading. Int $J$ Eng Sci, 49: 17-29, 2011.

[7] M. Hojo, Y. Matsushita, M. Tanaka, T. Adachi. In situ observation of interfacial crack propagation in GF/epoxy model composite using bifiber specimens in mode I and mode II loading. Compos Sci Technol, 68: 2678-2689, 2008.

[8] Z. Zou, S. Li. Stresses in an infinite medium with two similar circular cylindrical inclusions. Acta Mech, 156: 93-108, 2002.

[9] E. Correa, F. París and V. Mantič. Effect of the presence of a secondary transverse load on the inter-fibre failure under tension. Eng Fract Mech, 103: 174-189, 2013.

[10]F. París and J. Cañas. Boundary Element Method. Fundamentals and Applications. OUP, Oxford, 1997.

[11]A. Blázquez, F. París and V. Mantič. BEM solution of two-dimensional contact problems by weak application of contact conditions with non-conforming discretizations. Int $J$ Solids Struct, 35: 3259-3278, 1998.

[12]E. Graciani, V. Mantič, F. París and A. Blázquez. Weak formulation of axi-symmetric frictionless contact problems with boundary elements. Application to interface cracks. Comput Struct, 83: 836-855, 2005.

[13] G.R. Irwin. Analysis of stresses and strain near the end of a crack transversing a plate. $J$ App Mech, 24: 361-364, 1957.

[14] M. Toya. A crack along the interface of a circular inclusion embedded in an infinite solid. J Mech Phys Solids, 22: 325-348, 1974.

[15] Y. Murakami. Stress Intensity Factor Handbook. Pergamon Press, Oxford, 1988.

[16] J.W. Hutchinson and Z. Suo. Mixed mode cracking in layered materials. Adv App Mech, 29: 63-191, 1992.

[17] V. Mantič and F. París. Relation between SIF and ERR based measures of fracture mode mixity in interface cracks. Int. J. Fracture, 130: 557-569, 2004. 